\title{
Phytoprotection
}

\section{Les mycorhizes : un outil de protection des plantes mais non une panacée}

\section{Yolande Dalpé}

Volume 86, numéro 1, avril 2005

URI : https://id.erudit.org/iderudit/011715ar

DOI : https://doi.org/10.7202/011715ar

Aller au sommaire du numéro

\section{Éditeur(s)}

Société de protection des plantes du Québec (SPPQ)

\section{ISSN}

0031-9511 (imprimé)

1710-1603 (numérique)

Découvrir la revue

\section{Citer cet article}

Dalpé, Y. (2005). Les mycorhizes : un outil de protection des plantes mais non une panacée. Phytoprotection, 86(1), 53-59. https://doi.org/10.7202/011715ar
Résumé de l'article

Les mycorhizes arbusculaires constituent la symbiose végétale la plus répandue à l'échelle planétaire. Les champignons concernés, regroupés dans le phylum Glomeromycota et distribués sur l'ensemble des écosystèmes, colonisent la majorité des plantes terrestres. Aux avantages bien connus des mycorhizes sur la croissance végétale, s’ajoutent plusieurs bénéfices, notamment pour la survie des plantes, leur biodiversité, l'impact sur la microflore du sol et le potentiel d'agent de réduction des stress tant abiotiques que biotiques. Devant une telle panoplie d'avantages pour les plantes et l'environnement, on pourrait croire que les mycorhizes représentent une panacée à plusieurs problèmes liés à la production et à la protection des végétaux. Le complexe " plante-mycorhize-parasite-environnement » constitue la norme à maintenir ou à retrouver pour assurer la durabilité de l'environnement. Le fonctionnement des mycorhizes comme agent de lutte biologique touche globalement cinq mécanismes d'interaction. Certains concernent directement la plante, soit : 1) une stimulation de croissance par le biais d'un apport nutritif accru et une meilleure santé végétale, 2) une transformation morphologique au niveau racinaire, 3) l'induction ou la suppression de mécanismes de défense, notamment ceux impliquant plusieurs enzymes. D'autres agissent sur le parasite : 4) via une compétition directe avec les champignons mycorhiziens liée à la disponibilité de nutriments et de sites d'infection, et sur la structure et la qualité du sol, par le biais d'une 5) modification de la microflore et de l'augmentation du taux de matière organique.
Tous droits réservés @ La société de protection des plantes du Québec, 2005
Ce document est protégé par la loi sur le droit d'auteur. L'utilisation des services d'Érudit (y compris la reproduction) est assujettie à sa politique d'utilisation que vous pouvez consulter en ligne.

https://apropos.erudit.org/fr/usagers/politique-dutilisation/ 


\title{
Les mycorhizes : un outil de protection des plantes mais non une panacée
}

\author{
Yolande Dalpé ${ }^{1}$
}

PHYTOPROTECTION 86 : 53-59

\begin{abstract}
Les mycorhizes arbusculaires constituent la symbiose végétale la plus répandue à l'échelle planétaire. Les champignons concernés, regroupés dans le phylum Glomeromycota et distribués sur l'ensemble des écosystèmes, colonisent la majorité des plantes terrestres. Aux avantages bien connus des mycorhizes sur la croissance végétale, s'ajoutent plusieurs bénéfices, notamment pour la survie des plantes, leur biodiversité, l'impact sur la microflore du sol et le potentiel d'agent de réduction des stress tant abiotiques que biotiques. Devant une telle panoplie d'avantages pour les plantes et l'environnement, on pourrait croire que les mycorhizes représentent une panacée à plusieurs problèmes liés à la production et à la protection des végétaux. Le complexe "plante - mycorhize - parasite - environnement " constitue la norme à maintenir ou à retrouver pour assurer la durabilité de l'environnement. Le fonctionnement des mycorhizes comme agents de lutte biologique touche globalement cinq mécanismes d'interaction. Certains concernent directement la plante, soit: 1) une stimulation de croissance par le biais d'un apport nutritif accru et une meilleure santé végétale, 2) une transformation morphologique au niveau racinaire, 3) l'induction ou la suppression de mécanismes de défense, notamment ceux impliquant plusieurs enzymes. D'autres agissent sur le parasite: 4) via une compétition directe avec les champignons mycorhiziens liée à la disponibilité de nutriments et de sites d'infection, et sur la structure et la qualité du sol, par le biais d'une 5) modification de la microflore et de l'augmentation du taux de matière organique.
\end{abstract}

Mots clés: Lutte biologique mécanismes d'action, mycorhizes arbusculaires, symbiose.

\section{[Mycorrhizae: a potential tool for plant protection but not a panacea]}

Arbuscular mycorrhizae are the plant symbiosis the most widely spread on the planet. These fungi, grouped in the phylum Glomeromycota, are distributed over all terrestrial ecosystems and found associated with the majority of land plants. To the well-known positive impacts of arbuscular mycorrhizae on plant yields should be added several other benefits such as a better survival rate of colonized plants, the maintenance of plant biodiversity, the improvement of soil microflora, and the reduction in harmful effects of both biotic and abiotic environmental stresses. Given such a panoply of benefits to plants and their environment, one could believe that mycorrhizae represent a panacea for solving problems related to plant production and plant protection. In fact, the "plant - mycorrhizae - pathogen - environment" complex constitutes a standard condition to be maintained or to be recovered in order to ensure the sustainability of the environment. The potential of mycorrhizae as a biocontrol agent globally covers five known mechanisms of interaction. Three of them concern the direct effect of symbiosis on plants. They are: 1) plant growth stimulation through an increased nutritive contribution and, consequently, better plant health; 2) the morphological transformation of the root system; and 3) the induction or suppression of defense mechanisms, and this mainly at the enzymatic level. Another mechanism concerns the pathogen: 4) through a direct competition with mycorrhizal fungi linked with nutrient availability and infection sites. Finally, mycorrhizae indirectly influence the soil structure and quality through: 5) the modification of the soil microflora and an increase in organic matter.

Keywords: Action mechanisms, arbuscular mycorrhizae, biological control, symbiosis.

1. Agriculture et Agroalimentaire Canada, Ottawa (Ontario), Canada K1A 0C6; courriel : dalpey@agr.gc.ca 


\section{INTRODUCTION}

Une production végétale de qualité sous-entend des produits finis de haute gamme et l'utilisation de technologies qui préservent au mieux l'environnement. Toute approche réaliste visant à réduire l'apport de pesticides et de fertilisants chimiques et à protéger les cultures et la qualité des sols mérite d'être exploitée. L'association symbiotique entre des champignons du sol et les racines de la majorité des plantes terrestres entraîne un échange bidirectionnel de composés carbonés de la plante vers le symbiote fongique et de composés minéraux du champignon vers la plante. L'efficacité des fertilisants s'en voit améliorée, la croissance végétale stimulée, l'usage de pesticides atténué, et la résistance aux stress biotiques et abiotiques améliorée (Smith et Read 1997).

Les champignons mycorhiziens de type arbusculaire (MA), regroupés dans le phylum Glomeromycota, sont des champignons filamenteux coenocytiques qui colonisent à la fois le sol et les racines en établissant un pont entre la rhizosphère et la plante. Les hyphes extraracinaires augmentent le volume de sol (eau et minéraux) accessible à la plante alors que les hyphes intraracinaires se propagent dans les espaces intercellulaires, se gonflent en vésicules selon leur génotype et forment des arbuscules intracellulaires créant une importante zone interface entre les cellules des deux partenaires, sites privilégiés d'échanges nutritifs.

Le complexe "plante - mycorhize - environnement" constitue la norme dans la nature. Lors d'une exploitation intensive des sols cultivés via une fertilisation abondante et des traitements pesticides, production et protection végétales deviennent tributaires d'intrants chimiques et davantage susceptibles aux stress. Le potentiel des MA comme agents de lutte biologique a été répertorié chez des dizaines d'espèces cultivées en association avec plusieurs Gloméromycètes pour des affections d'origine principalement fongique et bactérienne. Cinq mécanismes d'action émergent de ces investigations, au niveau de la plante, du parasite et de la microflore du sol.

\section{MÉCANISMES D'ACTION}

Les premières investigations relatives au potentiel des MA comme agent de lutte biologique datent des années 1960 alors qu'on réalisait à peine l'impact bénéfique de ces organismes sur la croissance et la survie des plantes. Depuis, de nombreuses investigations permettent d'attribuer à ces organismes un rôle d'agent de lutte biologique (Singh et al. 2000; St-Arnaud et al. 1995; Whipps 2004; Xavier et Boyetchko 2002), qui s'interconnecte intimement à celui mieux connu de promoteur de croissance. Le grand nombre de plantes qui bénéficient du soutien des mycorhizes et la variabilité d'action des espèces et souches fongiques font que, lors de synthèses des travaux en lutte biologique effectués à ce jour, on doit sagement relativiser l'ensemble des résultats. Plusieurs des mécanismes d'action répertoriés jusqu'à maintenant agissent de manière indirecte sur les affections parasitaires. Ils concernent l'amélioration de la nutrition de la plante, la transformation de la morphologie végétale, la modification de la microflore et de la composition des sols. D'autres actions plus directes impliquent la stimulation de mécanismes de défense par la plante et la compétition entre mycorhizes et parasites pour l'espace, les sites d'infection et les éléments nutritifs. Dans tous les cas, les conditions de culture susceptibles d'influencer le développement des plantes, des parasites et des champignons mycorhiziens peuvent interférer sur le potentiel prophylactique des mycorhizes, qu'il s'agisse de facteurs abiotiques tels que la sécheresse, la température ou le taux de fertilisation du sol, ou de facteurs biotiques tels que les espèces végétales, mycorhiziennes et parasitaires en présence, l'état mycorhizien des plantes, la virulence des parasites et la diversité de la microflore compagne.

\section{Croissance et nutrition}

Connaissant le fonctionnement général des mycorhizes, l'augmentation de la croissance et de la santé générale des plantes colonisées se traduit par une vigueur accrue qui leur permet de mieux tolérer les stress environnementaux dont ceux causés par diverses maladies (Azcon-Aguilar et Barea 1996). Cette réduction de la susceptibilité aux infections n'est généralement efficace que lorsqu'une symbiose fonctionnelle s'établit préalablement à l'attaque du parasite car, dans la majorité des cas, ces derniers, une fois en contact avec leur hôte, envahissent nettement plus rapidement les tissus végétaux que les champignons mycorhiziens. Dans le cadre d'une analyse détaillée des résultats expérimentaux publiés disponibles, Pinochet et al. (1996) ont mis en évidence qu'effectivement il existe un facteur de cause à effet entre la nutrition améliorée des plantes mycorhizées et leur résistance aux attaques de parasites, mais que ce facteur ne compte que pour une partie seulement de la protection. Ainsi, dans le cas de la fusariose de la tomate, une nutrition optimale en phosphore n'atténue pas les symptômes de la maladie (Caron et al. 1986), ce qui indique clairement que d'autres mécanismes de protection viennent se greffer à la nutrition comme facteur de protection. De plus, les bénéfices de la mycorhization sur la protection contre les parasites apparaissent souvent une fois seulement que le parasite a envahi l'hôte, ce qui indique qu'une diversité de mécanismes sont impliqués dans la protection.

\section{Morphologie racinaire}

Chez certaines plantes, la colonisation mycorhizienne entraîne une ramification accrue du système racinaire, un raccourcissement des racines adventives, une augmentation des racines fines ou une transformation de l'architecture racinaire liée aux proportions respectives des catégories de racines. C'est le cas pour les espèces ligneuses : peuplier (Hooker et al. 1992), platane (Tisserant et al. 1991), prunier (Berta et al. 1995) et érable (Chapdelaine et al. 1998), ainsi que pour des plantes herbacées telles que le poireau (Berta et al. 1993), la tomate (Gamalero et al. 2004) et le fraisier (Norman et al. 1996). Dans ce dernier cas, la mycorhization s'accompagne d'une protection accrue contre la pourriture racinaire causée par Phytophthora fragariae Hickman alors qu'on pourrait croire qu'une abondance de ramifications puisse fournir à 
un tel parasite davantage d'opportunité pour infecter les tissus racinaires. La ramification intense des racines induite par la mycorhization s'accompagne d'une intensification des exsudats racinaires qui serait responsable de modifications de la microflore et d'interactions directes avec les parasites (Norman et Hooker 2000).

\section{Mécanismes de défense}

La colonisation mycorhizienne prédispose les plantes à réagir rapidement aux attaques de parasites (Dugassa et al. 1996; Singh et al. 2000). Cette protection indirecte se traduit au niveau cellulaire par des réactions anatomiques, métaboliques et physiologiques, ainsi que par l'induction ou la suppression de divers mécanismes de défense liés aux phytoalexines, phénols, peroxydases, chitinases, ß-glucanases, lignification, déposition de callose et diverses autres protéines liées à la pathogenèse. Les plantes colonisées produisent davantage d'éthylène, méthylent plus efficacement I'ADN et synthétisent davantage d'arginine dans leurs racines (Baltruschat et Schönbeck 1972; Harrier et Watson 2004), signes d'une hausse d'activités métaboliques.

On note chez certains couples "plante - mycorhize" une augmentation du taux de lignification des parois cellulaires de l'endoderme et des tissus vasculaires, et un dépôt de callose, ce qui peut expliquer la restriction des propagules mycorhiziennes au seul cortex racinaire. Cette lignification accrue constitue une barrière de protection pour la racine contre la pénétration de parasites et s'accompagne d'une accumulation de composés phénoliques vraisemblablement suivie d'une activité chitinolytique qui altère les parois, notamment de certains parasites fongiques (Benhamou et al. 1994; Sylvia et Chellemi 2001).

En ce qui a trait aux phytoalexines et aux gènes codant des processus de défense, leur induction varie selon les complexes plante - mycorhize - parasite (Harrison et Dixon 1993; Wyss et al. 1991); leur activité augmente généralement en début de colonisation pour disparaître une fois la symbiose établie. Le même scénario a été observé pour les enzymes phénylalanine ammonia - lyase (PAL) et chalcone synthase respectivement associées aux voies phénylpropanoïdes et à la synthèse de flavonoïdes et isoflavonoïdes (Lambais et Mehdy 1993; Volpin et al. 1994). Les phytoalexines peuvent être induites à la fois localement chez des cellules colonisées par les arbuscules mais aussi dans les tissus racinaires non colonisés.

L'activité des peroxydases et l'accumulation de phénols mises en place lors d'une infection parasitaire se retrouvent généralement inhibées au fur à mesure de l'établissement de la colonisation mycorhizienne, mais ne sont jamais détectées au niveau des cellules corticales habitées d'arbuscules (Spanu et Bonfante-Fasolo 1988), alors qu'elles peuvent être localement stimulées dans l'épiderme de la plante (Gianinazzi et Gianinazzi-Pearson 1992).

Les enzymes hydrolytiques chitinase et ß-glucanase jouent un rôle de protection, étant impliquées dans la dégradation des parois cellulaires. A ce titre, elles sont reconnues pour leur activité antifongique (Dumas-Gaudot et al. 1996). Une hausse de l'activité des chitinases et ß-glucanases a été observée chez les racines de légumineuses avant même tout contact racinaire avec Glomus intraradices Schenck \& Smith (Volpin et al. 1994) ainsi qu'au début de la colonisation, pour ensuite diminuer et pratiquement disparaître une fois la mycorhization établie et fonctionnelle (Lambais et Mehdy 1993; Spanu et al. 1989). Cette activité est d'ailleurs principalement liée aux jeunes arbuscules dont la longévité n'atteint que 3-4 jours (Blee et Anderson 1996). La dégradation continuelle des arbuscules intraracinaires entraîne une augmentation d'activité chitinolytique dans les couches profondes des cellules corticales ce qui peut directement affecter l'intégrité cellulaire des parasites (Benhamou et al. 1994; Dehne et al. 1978). La présence d'isoformes de chitinase nouvellement synthétisées et différentes de celles synthétisées par les parasites ont été détectées dans des racines de tabac et de tomates mycorhizées et parasitées (Cordier et al. 1996; Dumas-Gaudot et al. 1992), un mécanisme de défense spécifique aux parasites induit par les parois cellulaires de l'hôte (Pozo et al. 1998). En résumé, une colonisation mycorhizienne bien établie entraîne des changements considérables des mécanismes de défense de la plante pour contrer l'action de certains parasites (Guenoune et al. 2001; Wyss et al. 1991).

\section{Interactions mycorhize - parasite}

Lors d'infections parasitaires diverses, on note que dans $50 \%$ des cas les MA réduisent la croissance de parasites, alors que dans $32 \%$ des cas elles suppriment leur action néfaste et dans seulement $16 \%$ des cas le développement des mycorhizes est négativement affecté par le parasite (Borowicz 2001; Pinochet et al. 1996). L'interprétation de ces statistiques doit toutefois se faire prudemment. L'échantillonnage disponible à ce jour ne couvre que quelques dizaines de plantes cultivées en milieu agricole associées à une douzaine de souches mycorhiziennes testées sur un nombre encore limité de parasites affectant principalement les racines.

II est reconnu que le pouvoir protecteur des mycorhizes dépend de la nature du parasite (bactérie, champignon, virus) et diminue d'efficacité proportionnellement à l'augmentation de la virulence du parasite et à la concentration des propagules de ce dernier, tout en demeurant tributaire des conditions environnementales et de la nature des intervenants en présence. De plus, une réduction de l'incidence d'une maladie peut dépendre d'une résistance accrue de la plante et la baisse d'activité mycorhizienne peut impliquer une compétition directe des antagonistes pour les éléments nutritifs et la disponibilité des sites d'infection (Muchovej et al. 1991; Smith et GianinazziPearson 1988; Traquair 1995).

Les champignons MA, tout comme la plupart des parasites végétaux, et particulièrement les agents pathogènes fongiques, obtiennent leurs ressources nutritives carbonées directement de l'hôte végétal. Leur cohabitation avec la plante entraîne une compétition directe entre symbiote et parasite pour ces ressources énergétiques. Dans plusieurs cas, la croissance des deux organismes s'en trouve atténuée par une baisse du taux de sporulation du parasite ou par une réduction du taux de colonisation mycorhizienne des racines sans toutefois que les symptômes ou la 
sévérité de la maladie ne soient affectés. Dans cet ordre d'idée, Larsen et Bodker (2001) ont utilisé comme mesure de référence les taux respectifs d'acides gras neutres dans le parasite et le champignon mycorhizien au cours du développement de la pourriture racinaire de plants de pois causée par Aphanomyces euteiches Dreschler. Leurs résultats indiquent une perte énergétique réciproque chez les deux microorganismes ce qui suggère une compétition directe pour les sources carbonées.

Un modèle de prédiction de protection des plantes par les MA proposé par Smith et Gianinazzi-Pearson (1988) présente I'hypothèse qu'un parasite strictement obligatoire serait davantage affecté par les mycorhizes que des parasites capables d'un certain degré de saprophytisme (ex. rouille vs Fusarium). Le tout est basé sur plusieurs exemples montrant que les MA agissent sur la physiologie de la plante et rivalisent avec le parasite pour les ressources carbonées d'origine végétale et pour la disponibilité des sites d'infection. En fait, les quelques études effectuées sur l'interaction rouille-mycorhize montrent effectivement une augmentation de la biomasse végétale et une augmentation de l'incidence de la maladie (Meyer et Dehne 1986).

Dans une racine fortement colonisée par des souches mycorhiziennes à potentiel mycorhizien élevé, les hyphes colonisant les racines saturent davantage les sites d'infection disponibles, limitant ainsi la pénétration de la racine par les hyphes d'un parasite. Ceci ralentit ou retarde le développement du parasite et diminue d'autant l'incidence de la maladie. Une action systémique peut aussi être observée alors que l'incidence de la maladie est augmentée dans les zones racinaires non mycorhizées, la plante conservant intactes les zones colonisées. Dans le cas de virus, l'incidence de la maladie a plutôt tendance à augmenter chez les plantes mycorhizées (St-Arnaud et al. 1995). Par le biais de techniques d'immunofluorescence, il a été démontré que les virus se concentrent de préférence dans les cellules colonisées par les arbuscules, sites d'activité métabolique élevée. Le virus y bénéficie des échanges intensifs de minéraux, de protéines et d'acides nucléiques entre la plante et ses symbiotes mycorhiziens.

Les observations de St-Arnaud et al. (1995) et de Filion et al. (1999), obtenues in situ dans un système de culture monoxénique à deux compartiments, révèlent que le mycélium mycorhizien de $G$. intraradices stimule la germination prématurée des conidies de Fusarium oxysporum f. sp. chrysanthemi Littrell Armstr. \& Armstr. en absence de racines, sans détection d'antibiose. Extrapolées en milieu naturel, ces observations supposent un contrôle à distance d'infections parasitaires par le biais des hyphes extraracinaires.

\section{Microflore du sol}

L'activité microbienne des sols contribue considérablement à sa fertilité par le biais de synergies entre microorganismes, de compétitions et de parasitismes. Les hyphes extraracinaires des mycorhizes à arbuscules peuvent constituer à eux seuls jusqu'à $80 \%$ de la masse microbienne avec près de $150 \mathrm{~cm}$ $\mathrm{d}^{\prime}$ hyphes $\mathrm{cm}^{-3}$ de sol (Kabir et al. 1997). Une manipu- lation appropriée de cette microflore ou l'introduction d'organismes antagonistes aux parasites peut prévenir sinon contrôler la sévérité d'une infection (Linderman 1992). Bien que souvent négligée comme élément du complexe plante - mycorhize - parasite, cette microflore influence l'environnement physicochimique de la rhizosphère et contrôle diverses interactions microbiotiques du sol. Des nombreuses composantes microbiennes de la rhizosphère, certaines fonctionnent en synergie avec les plantes mycorhizées, favorisant croissance et protection alors que d'autres peuvent interférer négativement (Barea et al. 2002; Gryndler et al. 2000).

Ainsi, la mycorhization affecte directement la quantité et la qualité des exsudats racinaires, ce qui entraîne une réorganisation de la microflore de la rhizosphère, le tout tributaire des espèces fongiques et végétales en symbiose, de la maturation des plantes, de leur degré de mycorhization et des conditions environnantes (Bansal et al. 2000; Marschner et al. 2004; Meyer et Linderman 1986). L'activité mycorhizienne se traduit généralement par une augmentation de la diversité et de l'abondance des microorganismes du sol, notamment parmi les antagonistes de parasites (Linderman 2000; Secilia et Bagyaraj 1987; Thomas et al. 1994). Ces modifications de la microflore varient selon les paramètres éco-physiologiques et les souches mycorhiziennes testées, et peut affecter I'ensemble de la microflore ou encore l'une ou l'autre de ses composantes (Andrade et al. 1997). Ainsi, Meyer et Linderman (1986) ont mesuré dans la rhizosphère du maïs et du trèfle mycorhizés une augmentation de bactéries anaérobiques, une diminution des Pseudomonas, des actinomycètes et des Streptomyces, mais aucun changement chez des bactéries Gram négatif. Gryndler et al. (1995) ont observé en culture hydroponique une augmentation significative du nombre d'arbuscules lors de l'addition de bactéries capables de solubiliser des composés à base de phosphate ou de chitine.

La synergie entre les bactéries solubilisatrices de phosphate et les champignons MA se traduit par une stimulation de la germination des spores, de la colonisation racinaire mycorhizienne et par l'augmentation des populations bactériennes totales (Azcon-Aguilar et Barea 1995). Ces interactions représentent autant de possibilités de ralentir ou d'inhiber l'attaque de parasites.

II a également été mis en évidence que les mycorhizes peuvent stimuler l'exsudation d'antibiotiques par les racines et les bactéries du sol (Barea et al. 2002). Contrairement aux pesticides qui possèdent généralement un large spectre d'action envers la microflore, les multiples implications des mycorhizes dans la rhizosphère peuvent modeler la microflore microbienne de façon à remédier au déséquilibre causé par la prolifération excessive d'un parasite.

Certaines bactéries du sol secrètent des métabolites capables d'altérer la perméabilité des parois des cellules racinaires, d'en augmenter l'exsudation, de stimuler le développement des symbiotes mycorhiziens et la colonisation des racines (Jeffries et al. 2003). D'autre part, il a été démontré qu'une mycorhize fonctionnelle peut entraîner des variations importantes de la composition des exsudats racinaires. Les 
exsudats racinaires de tomates mycorhizées indiquent une hausse significative des taux d'acide aspartique et glutamique et une diminution des taux $\mathrm{d}^{\prime}$ arginine, de glycine et de thréonine (Harrier et Watson 2004). Il en résulte une légère baisse du pH du sol probablement suffisante pour retarder la sporulation ou ralentir le métabolisme de plusieurs parasites (Norman et Hooker 2000).

Certaines composantes de cette microflore interagissent avec les MA à la manière d'agents de lutte biologique. C'est le cas du Gliocladium virens Mill. et du Bacillus subtilis (Ehrenberg) Cohn qui, lorsqu'appliqués à une plante colonisée parasitée, stimulent à la fois la colonisation racinaire et réduisent l'incidence de la maladie (Bochow et Abou-Shaar 1990; Paulitz et Linderman 1991). En ce sens, plusieurs inoculants mycorhiziens commerciaux sont maintenant offerts en combinaison avec un agent de lutte biologique reconnu afin d'exploiter au mieux les pouvoirs conjoints de stimulateurs de croissance et $\mathrm{d}^{\prime}$ agent de lutte biologique.

\section{Statut général et perspectives}

La plupart des investigations présentées ci-dessus ont été menées en milieu contrôlé (chambres de croissance, serres ou parcelles choisies), combinant généralement un seul organisme parasite et une seule souche de champignon mycorhizien, les deux généralement reconnus respectivement pour leur potentiel pathogène et mycorhizien élevé. Le contexte de telles études ne reproduit aucunement les conditions de culture qui prévalent en champ ou en milieu naturel. L'extrapolation de telles données demeure hasardeuse compte tenu de la variabilité des résultats et de la différence de conditions qui sévissent en milieu naturel comparativement aux conditions d'expérimentation. Selon les souches mycorhiziennes et les cultivars de plantes étudiées, les résultats peuvent varier d'une protection à une aggravation de la maladie. De plus, les habiletés prophylactiques d'une souche donnée demeurent tributaires des conditions nutritives du sol, le taux de $\mathrm{P}$ disponible étant le facteur le plus influent connu à ce jour. L'expérimentation in situ sur le potentiel bioprotecteur des mycorhizes est donc ardemment requise afin de passer le plus rapidement et le plus efficacement possible à un usage plus fréquent et éventuellement une protection plus "douce" des cultures, et qui plus est, efficace sur une longue période.

Bien que restreint, le nombre de maladies et de plantes étudiées à ce jour permet de dire que les MA constituent une méthode de prévention prometteuse, facile d'utilisation et respectueuse de l'environnement. À ces atouts s'ajoute leur potentiel très bien répertorié d'organismes bénéfiques à la croissance, dont l'usage permet de réduire substantiellement l'apport externe d'intrants chimiques, de fertilisants et de pesticides.

L'usage des mycorhizes en agriculture doit être considéré comme une stratégie proactive à la lutte biologique. Connaissant l'impact conjugué des mycorhizes sur le rendement des cultures et comme agent de lutte biologique, leur introduction systématique dans les régies de production visent l'obtention de bénéfices relatifs à la fois à la production et à la protection des végétaux. La restauration du complexe "plante - mycorhize" dans les sols, soit par inoculation directe ou par transplantation de plantes prémycorhizées, permettrait de rétablir et surtout de conserver à long terme cet équilibre et ce potentiel pour la production et la protection des cultures. De plus, I'usage des mycorhizes s'accommode tout à fait des stratégies proactives déjà pratiquées pour certaines productions, tels que la rotation des cultures et le labour minimal. Une fois rétabli, le potentiel mycorhizien d'un sol peut perdurer durant de nombreuses années sans apport excessif ou onéreux de fertilisants et de pesticides, ce qui entraîne à moyen terme une économie non négligeable du coût de production, une meilleure conservation des terres, de la qualité des cultures et une réduction de l'usage de pesticides.

\section{RÉFÉRENCES}

Andrade, G., K.L. Mihara, R.G. Linderman et G.J. Bethlenfalvay. 1997. Bacteria from rhizosphere and hyphosphere soils of different arbuscular-mycorrhizal fungi. Plant Soil $192: 71-79$.

Azcon-Aguilar, C. et J.M. Barea. 1995. Saprophytic growth of arbuscular-mycorrhizal fungi. Pages 391-407 in B. Hock et A. Varma (éds.), Mycorrhiza structure function, molecular biology and biotechnology. Springer-Verlag, Heidelberg.

Azcon-Aguilar, C. et J.M. Barea. 1996. Arbuscular mycorrhizas and biological control of soil-borne plant pathogens - an overview of the mechanisms involved. Mycorrhiza 6 : 457-464.

Baltruschat, H. et F. Schönbeck. 1972. Influence of the endotrophic mycorrhiza on the chlamydospore production of Theilaviopsis basicola in tobacco roots. Phytopathol. Z. 4 : 358-361.

Bansal, M., N. Chamola, N. Sarwar et K.G. Mukerji. 2000. Mycorhizosphere: interaction between rhizosphere microflora and VAM fungi. Pages 143-152 in K.G. Mukerji, B.P. Chamola et J. Singh (éds.), Mycorrhizal biology. Kluwer Academic/Plenum Publishers, New York.

Barea, J.M., M. Toro, M.O. Orozco, E. Campos et R. Azcon. 2002. The application of isotopic (32P and $15 \mathrm{~N})$ dilution techniques to evaluate the interactive effect of phosphate-solubilizing rhizobacteria, mycorrhizal fungi and Rhizobium to improve the agronomic efficiency of rock phosphate for legume crops. Nutr. Cycl. Agroecosyst. $63: 35-42$.

Benhamou, N., J.A. Fortin, C. Hamel, M. St-Arnaud, et A. Shatilla. 1994. Resistance responses of mycorrhizal $\mathrm{Ri}$ T-DNA-transformed carrot roots to infection by Fusarium oxysporum f. sp. chrysanthemi. Phytopathology 84 : 958-968.

Berta, G., A. Fusconi et A. Trotta. 1993. VA mycorrhizal infection and the morphology and function of root systems. Environ. Exp. Bot. 33 : 159-173.

Berta, G., A. Trotta, A. Fusconi, J.E. Hooker, M. Munro, D. Atkinson, M. Giovannetti, S. Morini, P. Fortuna, B. Tisserant, V. Gianinazzi-Pearson et S. Gianinazzi. 1995. Arbuscular mycorrhizal induced changes to plant growth and root system morphology in Prunus cerasifera. Tree Physiol. 15 : 281-293.

Blee, K.A. et A.J. Anderson. 1996. Defense-related transcript accumulation in Phaseolus vulgaris L. colonized by the arbuscular mycorrhizal fungus Glomus intraradices Schenck and Smith. Plant Physiol. 110 : 675-688.

Bochow, H. et M. Abou-Shaar. 1990. On the phytosanitary effect of mycorrhiza in tomatoes to the corky-root disease. Z. Mikrobiol. 145 : 171-176.

Borowicz, V.A. 2001. Do arbuscular mycorrhizal fungi alter plant-pathogen relations? Ecology 82 : 3057-3068. 
Caron, M., J.A. Fortin et C. Richard. 1986. Effect of phosphorous concentration and Glomus intraradices on Fusarium crown and root rot of tomatoes. Phytopathology 76 : 942-946.

Chapdelaine, A., Y. Dalpé, C. Hamel, M. St-Arnaud, S. Kosuta, M. Pezzente, P. Jutras et S. Parent. 1998. Endomycorrhizal inoculation improves the City of Montreal nursery tree seedling production. Second International Conference on Mycorrhizae (ICOM2) Uppsala, Sweden, July 1998. 41 pp.

Cordier, C., S. Gianinazzi et V. Gianinazzi-Pearson. 1996. Colonisation patterns of root tissues by Phytophthora nicotianae var. parasitica related to reduced disease in mycorrhizal tomato. Plant Soil 185 : 223-232.

Dehne, H.W., F. Schoenbeck et H. Baltruschat. 1978. The influence of endotrophic mycorrhiza on plant diseases. 3. Chitinase activity and the ornithine cycle. Z. Pflanzenk. Pflanzen. 85 : 666-678.

Dugassa, G.D., H. Vonalten et F. Schönbeck. 1996. Effects of arbuscular mycorrhiza (AM) on health of Linum usitatissimum L. infected by fungal pathogens. Plant Soil 185 173-182.

Dumas-Gaudot, E., V. Furlan, J. Grenier et A. Asselin. 1992. New acidic chitinase isoforms induces in tobacco roots by vesicular-arbuscular mycorrhizal fungi. Mycorrhiza 1 : 133-136.

Dumas-Gaudot, E., S. Slezack, B. Dassi, M.J. Pozo, V. Gianinazzi-Pearson et S. Gianinazzi. 1996. Plant hydrolytic enzymes (chitinases and $\beta-1-3$ glucanases) in root reactions to pathogenic and symbiotic microorganisms. Plant Soil $185:$ 211-221.

Filion, M., M. St-Arnaud et J.A. Fortin. 1999. Direct interaction between the arbuscular mycorrhizal fungus Glomus intraradices and different rhizosphere microorganisms. New Phytol. $141: 525-533$.

Gamalero, E., A. Trotta, N. Massa, A. Copetta, M.G. Martinotti et G. Berta. 2004. Impact of two fluorescent pseudomonads and an arbuscular mycorrhizal fungus on tomato plant growth, root architecture and $\mathrm{P}$ acquisition. Mycorrhiza 14:185-192.

Gianinazzi, S. et V. Gianinazzi-Pearson. 1992. Cytology, histochemistry and immunocytochemistry as tools for studying structure and function in endomycorrhiza. Pages 109-139 in J.R. Norris, D.J. Read et A.K. Varma (éds.), Techniques for the study of mycorrhiza. (Methods in microbiology vol. 24). Academic Press, London.

Gryndler, M., H. Vejsacova, M. Vosatka et V. Catska. 1995 Influence of bacteria on vesicular-arbuscular mycorrhiza infection of maize. Folia Microbiol. 40 : 95-99.

Gryndler, M., H. Hrselová et D. Stríteská. 2000. Effect of soil bacteria on growth of hyphae of the arbuscular mycorrhizal (AM) fungus Glomus claroideum. Folia Microbiol. $45: 545-551$

Guenoune, D., S. Galili, D.A. Philips, H. Volpin, I. Chet, Y. Okon et Y. Kapulnik. 2001. The defense response elicited by the pathogen Rhizoctonia solani is suppressed by colonization of the AM-fungus Glomus intraradices. Plant Sci. $160: 925-932$.

Harrier, L.A. et C.A. Watson. 2004. The potential role of arbuscular mycorrhizal (AM) fungi in the bioprotection of plants against soil-borne pathogens in organic and/or other sustainable farming systems. Pest Manag. Sci. 60 : 149-157.

Harrison, M.J. et R.A. Dixon. 1993. Isoflavonoid accumulation and expression of defense gene transcripts during the establishment of vesicular-arbuscular mycorrhizal associations in roots of Medicago truncatula. Mol. PlantMicrobe Interact. $6: 643-654$

Hooker, J.E., M. Munro et D. Atkinson. 1992. Vesiculararbuscular mycorrhizal fungi induces alteration in poplar root system morphology. Plant Soil $145: 207-214$

Jeffries, P., S. Gianinazzi, S. Perotto, K. Turnau et J.M. Barea. 2003. The contribution of arbuscular mycorrhizal fungi in sustainable maintenance of plant health and soil fertility. Biol. Fertil. Soils $37: 1-16$.
Kabir, Z., I.P. O'Halloran, J.W. Fyles et C. Hamel. 1997. Seasonal changes of arbuscular mycorrhizal fungi as affected by tillage practices and fertilization: hyphal density and mycorrhizal root colonization. Plant Soil 192 : 285-293.

Lambais, M.R. et M.C. Mehdy. 1993. Suppression of endochitinase, ß-1,3-endoglucanase and chalcone isomerase expression in bean vesicular-arbuscular mycorrhizal roots under different soil phosphate conditions. Mol. Plant-Microbe Interact. $6:$ 75-83.

Larsen, J. et L. Bodker. 2001. Interactions between pea rootinhabiting fungi examined using signature fatty acids. New Phytol. 149 : 487-493.

Linderman, R.G. 1992. Vesicular-arbuscular mycorrhizae and soil microbial interactions. Pages 1-26 in G.J. Bethlenfalvay et R.G. Linderman (éds.), Mycorrhizae in sustainable agriculture. (ASA special publication) ASA Madison, WI, USA.

Linderman, R.G. 2000. Effects of mycorrhizas on plant tolerance to diseases. Pages 345-365 in Y. Kapulnik et D.D.Jr. Douds (éds.), Arbuscular mycorrhizas: physiology and function. Kluwer Academic, Dordrecht, The Netherlands.

Marschner, P., D. Crowley et C.H. Yang. 2004. Development of specific rhizosphere bacterial communities in relation to plant species, nutrition and soil type. Plant Soil 261: 199-208.

Meyer, J.M. et H.W. Dehne. 1986. The influence of VA mycorrhizae on biotrophic leaf pathogens. Pages 781-786 in V. Gianinazzi-Pearson et S. Gianinazzi (éds.), Physiological and genetical aspects of mycorrhizae. $1^{\text {st }}$ European Symposium on Mycorrhizae, INRA, Paris.

Meyer, J.R. et R.G. Linderman. 1986. Response of subterranean clover to dual inoculation with vesicular arbuscular mycorrhizal fungi and a plant growth-promoting bacterium Pseudomonas putida. Soil Biol. Biochem. 18 : 185-190.

Muchovej, J.J., R.M.C. Muchovej et E.J. Goncalves. 1991. Effect of kind and method of fungicidal treatment of bean seed on infections by the VA mycorrhizal fungus Glomus macrocarpum and by the pathogenic fungus Fusarium solani. Plant Soil 132: 47-51.

Norman, J.R. et J.E. Hooker. 2000. Sporulation of Phytophthora fragariae shows greater stimulation by exudates of non-mycorrhizal than by mycorrhizal strawberry roots. Mycologia 104 : 1069-1073.

Norman, J.R., D. Atkinson et J.E. Hooker. 1996. Arbuscular mycorrhizal fungal-induced alteration to root architecture in strawberry and induces resistance to the root pathogen Phytophthora fragariae. Plant Soil 185 : 191 198.

Paulitz, T.C. et R.G. Linderman. 1991. Lack of antagonism between the biocontrol agent Gliocladium virens and vesicular arbuscular mycorrhizal fungi. New Phytol. 117 : 303-308.

Pinochet, J., C. Calvet, A. Campruby et C. Fernandez. 1996. Interactions between migratory endoparasitic nematodes and arbuscular mycorrhizal fungi in perennial crops: a review. Plant Soil 185 : 183-190.

Pozo, M.J., C. Azcon-Aguilar, E. Dumas-Gaudot et J.M. Barea. 1998. Chitosanase and chitinase activities in tomato roots during interactions with arbuscular mycorrhizal fungi or Phytophthora parasitica. J. Exp. Bot. 49 : 1729-1739.

Secilia, J. et D.J. Bagyaraj. 1987. Bacteria and actinomycetes associated with pot cultures of vesicular-arbuscular mycorrhizas. Can. J. Microbiol. 33 : 1069-1073.

Singh, R., A. Adholeya et K.G. Mukerji. 2000. Mycorrhiza in control of soil-borne pathogens. Pages 173-196 in K.G. Mukerji, B.P. Chamola et J. Singh (éds.), Mycorrhizal biology. Kluwer Academic/Plenum Publishers, New York.

Smith, S.E. et V. Gianinazzi-Pearson. 1988. Physiological interactions between symbionts in vesicular-arbuscular mycorrhizal plants. Annu. Rev. Plant Physiol. Plant Mol. Biol. 39 : 221-244.

Smith, S.E. et D.J. Read. 1997. Mycorrhizal symbiosis. Academic Press, San Diego. 607 pp. 
Spanu, P. et P. Bonfante-Fasolo. 1988. Cell-wall-bound peroxidase activity in roots of mycorrhizal Allium porrum. New Phytol. 109 : 119-124.

Spanu, P., T. Boller, A. Ludwig, A. Wiemken, A. Faccio et P. Bonfante-Fasolo. 1989. Chitinase in roots of mycorrhizal Allium porrum: regulation and localization. Planta 177 : 447-455.

St-Arnaud, M., C. Hamel, M. Caron et J.A. Fortin. 1995. Endomycorhizes VA et sensibilité des plantes aux maladies : synthèse de la littérature et mécanismes d'interaction potentiels. Pages 51-87 in J.A. Fortin, C. Charest et Y. Piché (éds.), La symbiose mycorhizienne. État des connaissances. Editions Orbis Publishing, Frelisghburg, Québec.

Sylvia, D.M. et D.O. Chellemi. 2001. Interactions among root-inhabiting fungi and their implications for biological control of root pathogens. Adv. Agron. $73: 1-33$.

Thomas, L., B.C. Mallesha et D.J. Bagyaraj. 1994. Biological control of damping-off of cardamom by the VA mycorrhizal fungus, Glomus fasciculatum. Microbiol. Res. 149 : 413-417.

Tisserant, B., L. Schellenbaum, V. Gianinazzi-Pearson, S. Gianinazzi et G. Berta. 1991. Influence of infection by an endomycorrhizal fungus on root development and architecture in Platanus acerifolia. Allionia 30 : 171-181.
Traquair, J.A. 1995. Fungal biocontrol of root diseases: endomycorrhizal suppression of Cylindrocarpon root rot. Can. J. Bot. 73 : S89-S95.

Volpin, H., Y. Elkind, Y. Okon et Y. KapulnikY. 1994. A vesicular arbuscular mycorrhizal fungus (Glomus intraradix) induces a defense response in alfalfa roots. Plant Physiol. $104: 683-689$.

Whipps, J.M. 2004. Prospects and limitations for mycorrhizas in biocontrol of root pathogens. Can. J. Bot. 82 : 1198-1227.

Wyss, P., T. Boller et A. Wiemken. 1991. Phytoalexin response is elicited by a pathogen (Rhizoctonia solani) but not by a mycorrhizal fungus (Glomus mosseae) in soybean roots. Experientia $47: 395-399$.

Xavier, L.J.C. et S.M. Boyetchko. 2002. Arbuscular mycorrhizal fungi as biostimulants and bioprotectants of crops. Appl. Mycol. Biotechnol. 2 : 311-340. 